\title{
Hueso Cortical en Sínfisis Mandibular de Sujetos de Clase I
}

\author{
Cortical Bone in Mandibular Symphysis of Class I Subjects
}

Humberto Velásquez ${ }^{*, * *}$; Sergio Olate ${ }^{*, * * * * * * * *}$; Mariano del Sol ${ }^{* * * * *}$; Mario Cantín ${ }^{* * *}$; Bélgica Vásquez ${ }^{* * * * * *}$

VELÁSQUEZ, H.; OLATE, S.; DEL SOL, M.; CANTIN, M. \& VÁSQUEZ, B. Hueso cortical en sínfisis mandibular de sujetos de Clase I. Int. J. Morphol., 34(1):371-374, 2016.

RESUMEN: El objetivo fue evaluar la relación de tejido óseo cortical presente en el sector anterior y posterior de sínfisis mandibular. Se seleccionaron 18 sujetos clase I dentaria y esqueletal, de ambos sexos, a partir de una tomografía computarizada de haz cónico para analizar morfométricamente en base a la posición de los dientes canino, incisivo lateral e incisivo central de ambos lados; se analizó la distancia vertical desde el ápice dentario hasta el punto más inferior del margen mandibular y la distancia anteroposterior en dos niveles inferiores del ápice (5 mm y $10 \mathrm{~mm}$ ), donde se identificó el grosor de hueso cortical. El análisis de datos fue realizado con la prueba ANOVA considerando un valor de $\mathrm{p}<0,05$. Se observó que el hueso cortical es de mayor tamaño en la cortical lingual que la cortical bucal con casi $1 \mathrm{~mm}$ de diferencia; la cortical inferior fue la de mayor tamaño; no se observaron diferencias en las mediciones realizadas para cada diente. Se concluye que el hueso cortical es de menor tamaño en el sector bucal al compararse con el lingual e inferior; se debe explorar los alcances quirúrgicos de este hallazgo.

PALABRAS CLAVE: Sínfisis mandibular; Morfología mandibular; Osteotomía mandibular.

\section{INTRODUCCIÓN}

La región anterior de mandíbula se relaciona con importantes condiciones anatómicas que permiten la función y la adecuada armonía facial. La inserción de músculos y la posición del labio inferior son claves en esta zona, así como también las condiciones dentarias relacionadas (Olate et al., 2014).

En casos de trauma facial, la sínfisis mandibular debe ser recuperada mediante osteosíntesis en diferentes niveles (Al-Moraissi \& Ellis, 2014); el tipo de fractura generada en esta zona puede ser variable y responde a condiciones como energía del trauma, presencia de dientes, condiciones óseas, entre otras.

Otras situaciones relacionadas a la sínfisis mandibular son las osteotomías que se emplean para realizar mentoplastía (Assis et al., 2014) o también para la toma de injertos (Nóia et al., 2011). En estas condiciones clínicas es necesario intervenir con fresas o sierras en diferentes áreas sectores de la sínfisis mandibular.
La manipulación de esta zona, por tanto, requiere el conocimiento y refinamiento anatómico a fin de estimar las condiciones precisas en las que se realizan estos procedimientos (Vásquez et al., 2015). La morbilidad asociada, por ejemplo, en la toma de injertos de sínfisis mandibular, es definitivamente mayor que en otras zonas intraorales (Nóia et al.).

El objetivo de la presente investigación fue conocer la condición morfológica de sínfisis mandibular en sujetos con características de clase I esqueletal.

\section{MATERIAL Y MÉTODO}

Se realizó un estudio descriptivo para analizar el hueso cortical en el sector bucal, lingual e inferior de sínfisis. Se incluyeron sujetos que presentaran clase I dento esqueletal definida en base a la clase I oclusal de Angle y que mantuvieran todos sus dientes a excepción de los terce-

\footnotetext{
* Programa de Magíster en Odontología, Facultad de Odontología, Universidad de La Frontera, Temuco, Chile.

** Facultad de Odontología, Universidad San Sebastián, Puerto Montt, Chile.

*** División de Cirugía Oral y Maxilofacial \& CIMA, Universidad de La Frontera, Temuco, Chile.

**** Centro de Investigación en Ciencias Biomédicas, Universidad Autónoma de Chile, Temuco, Chile

***** Programa de Doctorado en Ciencias Morfológicas, Universidad de La Frontera, Temuco, Chile.

******** Universidad de Tarapacá, Arica, Chile.
} 
ros molare superiores e inferiores. Se excluyeron sujetos que se hubiesen sometido a tratamientos endodónticos o tratamiento de ortodoncia en el sector mandibular, que hubiese presentado cirugías faciales, historia de trauma facial o presencia de quistes o tumores mandibulares.

El estudio se realizó analizando imágenes planas obtenidas de tomografía computarizada de haz cónico (TCCB) realizadas en posición de máxima intercuspidación utilizando el equipo PlanMeca ${ }^{\circledR}$ (Korea); las imágenes fueron analizadas en el programa computacional EZ3D plus (2009) por un observador único siguiendo protocolos de repetición de mediciones y análisis intra clase de rutina según el protocolo desarrollado.

Los puntos de medición se localizaron en relación a los dientes canino, incisivo lateral e incisivo central, del lado derecho e izquierdo en cada mandíbula; en todos estos dientes se plantearon 5 unidades de medición (expresadas en milímetros) de los cuales dos fueron posteriores, dos fueron anteriores y uno fue inferior (Fig. 1). Las mediciones horizontales se realizaron $5 \mathrm{~mm}$ hacia inferior del ápice del diente respectivo mientras que la medición inferior siguió la línea generada a partir del ápice dentario coincidiendo en ángulo recto con la línea horizontal.
Cada medida fue realizada en tres oportunidades por el mismo observador, estableciendo el análisis de error para posteriormente establecer las medidas y promedios desde el análisis de J e I y desde F y G. El análisis de datos se realizó con las pruebas estadísticas de ANOVA, considerando un valor de $\mathrm{p}<0,05$ para establecer significancia estadistica

\section{RESULTADOS}

Los resultados obtenidos se observan en la Tabla I. La cortical bucal presentó un promedio de tamaño de cortical de $1,67 \mathrm{~mm}( \pm 0,43 \mathrm{~mm})$; ya la cortical lingual presentó un tamaño promedio de $2,71 \mathrm{~mm}( \pm 0,89 \mathrm{~mm})$ lo que significa prácticamente $1 \mathrm{~mm}$ mas al comparar con en el área de cortical bucal.

En la cortical bucal, la mayor diferencia de tamaño se observó con 0,26 mm de diferencia, las cuales no fueron significativas cuando se compararon los dientes caninos, incisivos lateral e incisivo central derecho e izquierdo. La mayor diferencia encontrada en la cortical lingual fue de 0,27 mm cuando se realizó la misma comparación, demostrando consistencia en el tamaño de la cortical en todo el sector anterior de mandíbula.

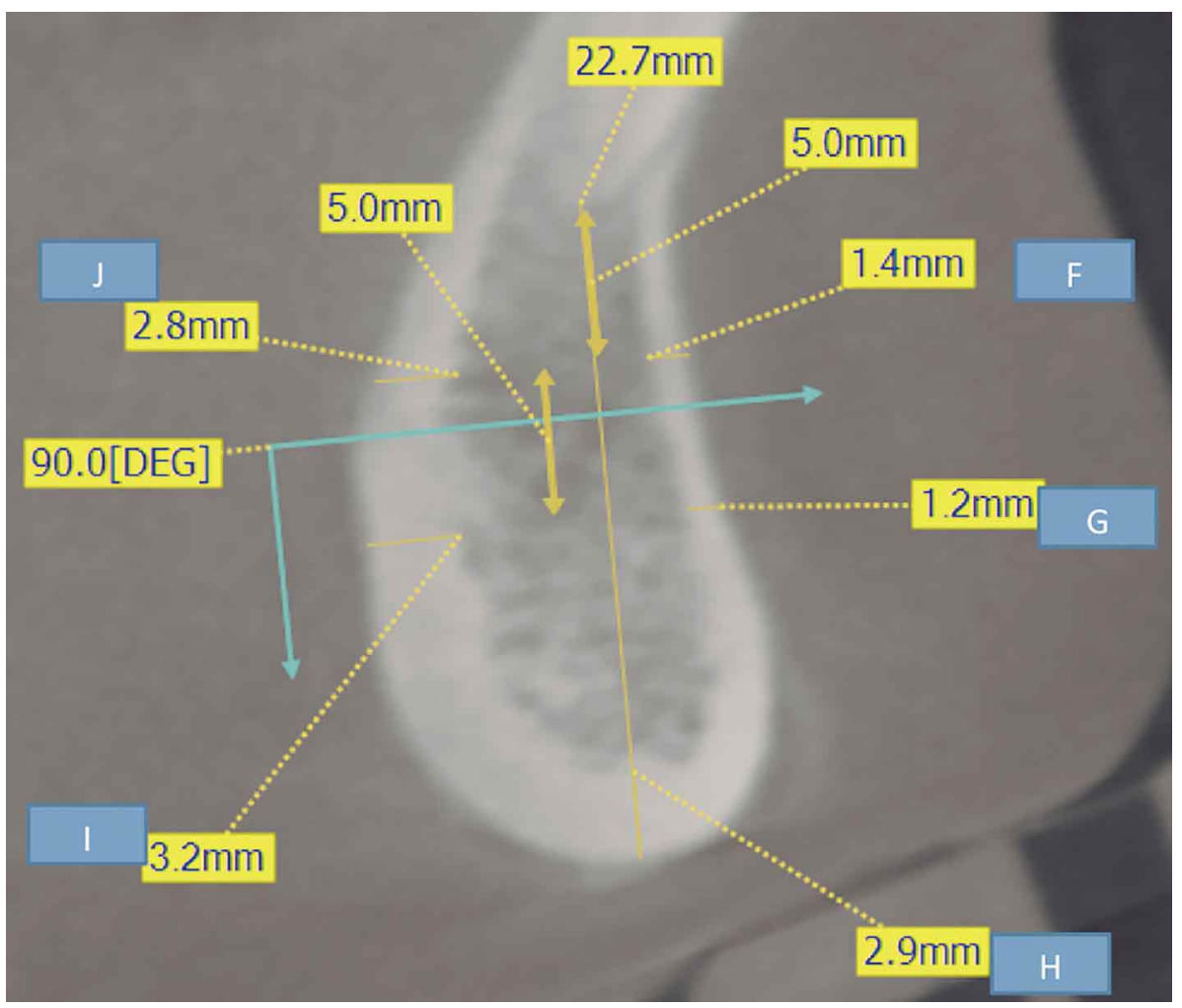

Consistentemente, en todas las mediciones realizadas a nivel dentario, la cortical lingual fue de mayor tamaño que la cortical bucal, presentando relaciones estadísticamente significativas $(P=0,000)$.

La zona inferior de la sínfisis presento un tamaño cortical de $3,01 \mathrm{~mm}( \pm 0,79 \mathrm{~mm})$, presentándose como el área cortical de mayor tamaño en la sínfisis; solamente a nivel de incisivos centrales esta cortical inferior presentó 2,66 y 2,42 mm; a nivel de caninos, la cortical inferior presentó tamaños de 3,57 y 3,37 mm. Esta área cortical fue la que presentó mayor variabilidad cuando se analizó la relación de cada diente.

Fig. 1. Mediciones realizadas en la región de sínfisis mandibular de 18 sujetos clase I esqueletal. 
Tabla I. Mediciones obtenidas en el análisis de cortical de sínfisis de 18 sujetos con relación esqueletal de clase I.

\begin{tabular}{llcccccc}
\hline \multicolumn{2}{c}{ Diente } & \multicolumn{2}{c}{ Cortical Bucal } & \multicolumn{2}{c}{ Cortical Lingual } & \multicolumn{2}{c}{ Cortical Inferior } \\
& & Promedio & DE & Promedio & DE & Promedio & DE \\
\hline Derecho & Canino & 1,65 & $\pm 0,50$ & 2,39 & $\pm 0,85$ & 3,57 & $\pm 1,31$ \\
& I. Lateral & 1,56 & $\pm 0,28$ & 2,69 & $\pm 0,81$ & 3,09 & $\pm 0,59$ \\
& I. Central & 1,68 & $\pm 0,53$ & 2,74 & $\pm 0,78$ & 2,66 & $\pm 0,70$ \\
Izquierdo & I. Central & 1,63 & $\pm 0,41$ & 2,96 & $\pm 1,11$ & 2,42 & $\pm 0,58$ \\
& I. Lateral & 1,59 & $\pm 0,37$ & 2,82 & $\pm 0,94$ & 3,00 & $\pm 0,70$ \\
& Canino & 1,91 & $\pm 0,54$ & 2,71 & $\pm 0,89$ & 3,37 & $\pm 0,89$ \\
Promedio & & 1,67 & $\pm 0,44$ & 2,72 & $\pm 0,90$ & 3,02 & $\pm 0,80$ \\
\hline
\end{tabular}

\section{DISCUSIÓN}

El uso de tomografía computadorizada cone beam ha sido utilizada consistentemente en el diagnostico y evaluación morfológica de diferentes áreas del esqueleto facial. Con esta tecnología, Pereira-Maciel et al. (2015), demostraron la presencia del canal incisivo en el $100 \%$ de la evaluaciones realizadas, señalando la necesidad del uso de estos exámenes para el análisis anatómico y estructural del área de sínfisis mandibular.

Nuestros resultados reportan la presencia de una consistente estructura cortical en el sector bucal, lingual e inferior de la región de sínfisis mandibular. El área bucal fue la de menor en tamaño, seguida de la lingual y la inferior. A pesar de que Garlock et al. (2016), indicaron que existen variables que pueden influenciar en las variaciones de tamaño de cortical como la posición dentaria y el ancho de reborde, nuestros resultados mostraron consistencia en las mediciones presentadas. La estructura cortical depende también de condiciones fisiológicas normales, donde Wang et al. (2016), realizando un estudio en modelo animal, señalaron que la deficiencia de testosterona en macacos machos influenciaría en la disminución de la estructura de hueso cortical y las consecuentes enfermedades de soporte dentario.

Diferencias entre sexo también fueron confirmadas por Atilli et al. (2015), quienes concluyeron que entre hombres y mujeres existía una diferencia de densidad mineral en la cortical bucal, en ausencia de diferencias en la cortical lingual, probablemente debido a la inserción muscular y función diaria que presentaba esta zona. Por otra parte, Lee et al. (2014) indicaron que el tamaño de la cortical de sínfisis tendría relaciones con el género del paciente pero que no tendría la influencia con la edad de los sujetos analizados.

Condiciones de tamaño cortical son necesarias de identificar para la práctica clínica diaria. Flanagan (2015), estudió el tiempo del efecto anestésico y su relación con el hueso cortical, indicando que sujetos con tamaño de cortical prome- dio de 2,12 mm presentaban sensibilidad al estimulo eléctrico luego de $5 \mathrm{~min}$ de aplicado la anestesia infiltrativa local mientras que sujetos con promedios de 2,0 mm no presentaban ningún tipo de sensibilidad en el mismo tiempo de evaluación, concluyendo que el tamaño de cortical era uno de los motivos para mayor o menor tiempo de acción del anestésico local.

Lee et al. (2015), realizaron una interesante investigación donde observaron sujetos con oclusión dentaria sin anomalías de posición; identificaron que a nivel del incisivo central el tamaño de cortical bucal fue de $1,8 \mathrm{~mm}$; a nivel de incisivo lateral, 2,0 $\mathrm{mm}$ y a nivel del canino, 2,3 $\mathrm{mm}$. Nuestros resultados no mostraron ningún tamaño de cortical bucal superior a $2 \mathrm{~mm}$; esta condición se puede asociar a diferencias en calidad de imagen o a la composición poblacional.

Los resultados de esta investigación están en relación con resultados previos (Vásquez et al., 2015). En sujetos edéntulos, Flanagan (2008) reportó que el hueso cortical lingual fue de 2,33 mm mientras que el bucal fue de 1,79 mm, notando diferencias de $1 \mathrm{~mm}$ entre ambos sectores. Kim et al. (2012) estudiaron cadáveres dentados concluyendo el mayor tamaño del hueso cortical lingual. Genisa et al. (2014) indicaron que en 10 sujetos evaluados con tomografías de haz cónico se observó que el hueso cortical lingual fue significativamente mayor al hueso cortical bucal, presentando diferencias de 1, $34 \mathrm{~mm}$ en el sector anterior y 2,92 en el sector posterior (1,58 $\mathrm{mm}$ de diferencia).

La importancia de conocer la morfología cortical, clínicamente, se establece en la gran cantidad de procedimientos quirúrgicos que se realizan en la zona y que han demostrado cierto grado de morbilidad asociada (Nóia et al.). Además de ello, la cortical inferior de mandíbula ha sido analizada como una zona capaz de caracterizar la evolución de patologías asociadas al uso de bifosfonatos donde Torres et al. (2015), indicaron que la altura de hueso cortical inferior de mandíbula era significativamente mayor en sujetos sometidos a tratamiento con bifosfonatos al ser comparado con controles y que este aumento era relacionado con dosis acumulativas de zeledronato. 
Se concluye que la cortical inferior y lingual son de mayor tamaño que la cortical bucal y que esta ultima presenta valores promedio de 1,6 mm de extensión. Nuevas consideraciones clínicas se deben realizar a partir de estos resultados.

VELÁSQUEZ, H.; OLATE, S.; DEL SOL, M.; CANTIN, M. \& VÁSQUEZ, B. Cortical bone in mandibular symphysis of Class I subjects. Int. J. Morphol., 34(1):371-374, 2016.

SUMMARY: The aim of this research was to evaluate the relation between the cortical bone in the anterior and posterior area of the mandible. Were selected 18 subjects, male and female, with dental and skeletal class I; in all of them was realized a cone beam computed tomography to make a morphometric analysis in agreed with de canine, lateral incisor and central incisor in the right and left, side. Was analyzed the distance in cortical area in a line from the dental apex and the horizontal line was obtained $5 \mathrm{~mm}$ and 10 $\mathrm{mm}$ from the dental apex; statistical significance was obtained by ANOVA considering a $p$ value $<0.05$. Was observed that the cortical bone is thicker in the lingual side than the buccal side $(1 \mathrm{~mm}$ diference approximately); the low cortical presented bigger size than anterior or posterior cortical bone; was not observed statistical differences between bone related to teeth. Its concluded that the buccal cortical bone is lower than lingual cortical bone; it is necessary an analysis about the surgical implications.

KEY WORDS: Mandible symphysis; Mandible morphology; Mandible osteotomy.

\section{REFERENCIAS BIBLIOGRÁFICAS}

Al-Moraissi, E. A. \& Ellis, E. Surgical Management of Anterior Mandibular Fractures: A Systematic Review and Meta-analysis. J. Oral Maxillofac. Surg., 72(12):2507.e1-11, 2014.

Assis, A.; Olate, S.; Asprino, L. \& de Moraes, M. Osteotomy and osteosynthesis in complex segmental genioplasty with doublé surgical guide. Int. J. Clin. Exp. Med., 7:1197-203, 2014.

Attilli, S.; Surapaneni, H.; Kasina, S. P.; Balusu, S. \& Barla, S. C. To Evaluate the Bone Mineral Density in Mandible of Edentulous Patients using Computed Tomography: An In Vivo Study. J. Int. Oral Health, 7:22-26, 2015.

Flanagan, D. A Comparison of facial and lingual cortical thicknesses in edentulous maxillary and mandibular sites measured on computerized tomograms. J. Oral Implantol., 34(5):256-8, 2008.

Flanagan, D. F. The effectiveness of articaine in mandibular facial infiltrations. Local Reg. Anesth., 9:1-6, 2015.

Garlock, D. T.; Buschang, P. H.; Araujo, E. A.; Behrenys, R. G. \& Kim, K. B. Evaluation of marginal alveolar bone in the anterior mandible with pretreatment and posttreatment computed tomography in nonextraction patients. Am. J. Orthod. Dentofacial. Orthop., 149(2):192-201, 2016.
Genisa, M.; Rajion, Z.; Pohchi, A.; Yap, J. \& Rafiq, M. Preoperative bone assessment using cone beam computed tomography. Int. Med. J., 21(4):412-5, 2014

Kim, H. J.; Yu, S. K.; Lee, M. H.; Lee, H. J.; Kim, H. J. \& Chung, C. H. Cortical and cancellous bone thickness on the anterior region of alveolar bone in Korean: a study of dentate human cadavers. $J$. Adv. Prosthodont., 4(3):146-52, 2012.

Lee, J. E.; Lee, Y. J.; Jin, S. H.; Kim, Y.; Kook, Y. A.; Ko, Y. \& Park, J. B. Topographic analysis of the mandibular symphysis in a normal occlusion population using cone-beam computed tomography. Exp. Ther. Med., 10(6): 2150-6, 2015.

Lee, K. A.; Kim, M. S.; Hong, J. Y.; Lee, J. S.; Choi, S. H.; Chai, J. K. \& Jung, U. W. Anatomical topography of the mandibular symphysis in the Korean population: a computed tomography analysis. Clin. Anat., 27:592-7, 2014.

Nóia, C. F.; Ortega-Lopes, R.; Olate, S.; Duque, T. M.; de Moraes, M. \& Mazzonetto, R. Prospective clinical assessment of morbidity after chin bone harvest. J. Craniofac. Surg., 22:2195-8, 2011.

Olate, S.; Cantín, M.; Vásquez, B.; del Sol, M. \& de Moraes, M. Facial Soft Tissue in Subjects with Class II and Class III Fa- cial Deformities: Preliminary Results. Int. J. Morphol., 32(3):1022-5, 2014.

Pereira-Maciel, P.; Tavares-de-Sousa, E. \& Oliveira-Sales, M. A. The mandibular incisive canal and its anatomical relationships: A cone beam computed tomography study. Med. Oral Patol. Oral Cir. Bucal, 20:e723-e728, 2015.

Torres, S. R.; Chen, C. S.; Leroux, B. G.; Lee, P. P.; Hollender, L. G.; Lloid, M.; Drew, S. P. \& Schubert, M. M. Mandibular inferior cortical bone thickness on panoramic radiographs in patients using bisphosphonates. Oral Surg. Oral Med. Oral Pathol. Oral Radiol., 119:584-92, 2015

Velásquez, H.; Olate, S.; del Sol, M.; Cantín, M. \& de Moraes, M. Osseous Morphology in the Mandibular Symphysis. APilot Study. Int. J. Morphol., 33(1):355-60, 2015.

Wang, Q.; Kessler, M. J.; Kensler, T. B. \& Dechow, P. C. The mandibles of castrated male rhesus macaques (Macaca mulatta): The effects of orchidectomy on bone and teeth. Am. J. Phys. Anthropol., 159:31-51, 2016.

Dirección para Correspondencia:

Prof. Sergio Olate

División de Cirugia oral y Maxilofacial

Claro Solar 115 oficina 414-A

Temuco, CHILE

Email: sergio.olate@ufrontera.cl 